\title{
Infants May Use Contingency Analysis to Estimate Environmental States: An Evolutionary, Life-History Perspective
}

\author{
Willem E. Frankenhuis, ${ }^{1}$ György Gergely, ${ }^{1}$ and John S. Watson ${ }^{2}$ \\ ${ }^{1}$ Central European University and ${ }^{2}$ University of California, Berkeley
}

ABSTRACT-Harshness and unpredictability early in life appear to be independently associated with long-term developmental outcomes, with environmental stressors affecting parental investment (e.g., responsiveness), which then shapes child development (e.g., onset of puberty). Research has detailed mediating physiological pathways, but has not specified how children transform experiences with their parents' and others' responsiveness into cognitive estimates about environmental harshness or unpredictability. In this article, we analyze this estimation process drawing on the study of contingency analysis. We propose that harshness and unpredictability produce distinct contingency profiles, and that children use these profiles to separately estimate each dimension. We conclude with six empirical predictions.

KEYWORDS—life-history development; social contingency; parenting; psychosocial acceleration; harshness; unpredictability; control

Willem E. Frankenhuis and György Gergely, Cognitive Development Center, Central European University, Hungary; John S. Watson, Department of Psychology, University of California, Berkeley, CA.

This research was supported by the Hungarian Science Foundation (OTKA NK 83997). We thank Jay Belsky, Andrew Delton, Bálint Forgács, Irene Godoy, Christophe Heintz, Mikolaj Hernik, and two anonymous reviewers.

Correspondence concerning this article should be addressed to Willem E. Frankenhuis, Cognitive Development Center, Central European University, Hattyú u. 14, 1015 Budapest, Hungary; e-mail: wfrankenhuis@gmail.com.

(C) 2013 The Authors

Child Development Perspectives $\odot 2013$ The Society for Research in Child Development DOI: $10.1111 /$ cdep.12024
Children use early experiences to guide their long-term development (Belsky, Houts, \& Fearon, 2010; Belsky, Steinberg, \& Draper, 1991; Chisholm, 1993; Chisholm, Quinlivan, Petersen, \& Coall, 2005; Ellis, 2004). Stressors in the environment affect parental investment (e.g., responsiveness), which then shapes children's development (e.g., attachment styles) into adolescence (e.g., onset of puberty) and adulthood (e.g., mating strategies). Research has detailed mediating physiological pathways (Cameron et al., 2008; Ellis, 2004), but has not specified how children transform experiences with their parents' and others' responsiveness into cognitive estimates about environmental harshness or unpredictability.

In this article, we analyze this estimation process drawing on the study of contingency analysis in humans (Gergely \& Watson, 1999; Tarabulsy, Tessier, \& Kappas, 1996; Watson, 1967, 1979, 2001) and other animals (Maier \& Seligman, 1976; Shanks, 1995). First we introduce life-history theory. Next we show that early-life harshness and unpredictability affect long-term development. Then we argue that children separately estimate their environment's level of harshness and predictability using contingency analysis (i.e., conditional probabilities of actions and outcomes). We conclude with six empirical predictions.

\section{LIFE-HISTORY DEVELOPMENT}

Life-history theory concerns organisms' allocations of time and energy across different fitness-relevant activities, including growth and development, mating, and parenting (Promislow \& Harvey, 1990; Stearns, 1992). The approach assumes that natural selection has shaped developmental mechanisms to make adaptive resource allocations depending on the local ecology (e.g., level of danger) and an individual's physical and social state (e.g., health, social network). For instance, in high-mortality environments, organisms might invest in early reproduction, which is likely adaptive when the risk of dying young is great (Belsky, 2012; Belsky et al., 1991; Belsky et al., 2010; 
Chisholm, 1993; Chisholm et al., 2005; Ellis, 2004; Nettle, Coall, \& Dickins, 2011).

Of key interest to life-history theorists is developmental plasticity - the ability to adjust developmental trajectories based on experience (Stearns, 1992). Developmental plasticity allows individuals to "tailor" their phenotypes to local environmental conditions, and can evolve by natural selection when the environmental state (e.g., safe or dangerous) varies across time or space (Ellis, Figueredo, Brumbach, \& Schlomer, 2009; Frankenhuis \& Del Giudice, 2012). Developmental plasticity can be viewed as a sampling process in which individuals obtain "cues"-features that tell them about the environmental state (e.g., high-quality parental care indicates a "safe" environment)that may guide their development (Frankenhuis \& Panchanathan, 2011a, 2011b).

\section{Resources, Mortality, and Unpredictability}

Drawing on biology, evolutionary psychologists have identified three key environmental dimensions affecting human life-history development: (a) resource availability, (b) harshness (i.e., extrinsic mortality-morbidity; unpreventable causes of disability and death), and (c) unpredictability (i.e., random variability in harshness over time; Ellis et al., 2009). Resource availability is fundamental because energy provides the building blocks for growth and development. In conditions of extreme energetic stress, individuals grow slowly, delay sexual maturation, develop small bodies, and produce few offspring (Ellison, 2001; although see Painter et al., 2008). Once minimal energetic demands are met, harshness and unpredictability can become major determinants of life-history development.

Environments are unpredictable for different reasons: Individuals might move from one environment to another (e.g., residential changes) or the environment itself may change (e.g., resource variability). Even if the environment remains the same, individuals may be unable to predict events that matter to them, in part because they cannot control them (e.g., chaotic households). These reasons are independent; for instance, an individual may move repeatedly between different environments, which themselves may be stable or unstable.

\section{Psychosocial Acceleration}

Psychosocial stress in early life influences reproductive development (Belsky, 2012; Ellis, 2004) and is associated with developmental trajectories oriented toward early reproduction, as evidenced by early puberty (Belsky et al., 1991; Belsky et al., 2010), early onset of sexual activity (Ellis et al., 2009), increased interest in infants (Maestripieri, Roney, De Bias, Durante, \& Spaepen, 2004), and early conception (Nettle et al., 2011). Although studies on this subject are often correlational, some work has used genetically informative designs (Tither \& Ellis, 2008) and experiments of nature (Pesonen et al., 2008). Direct experimental evidence from animals causally links adverse early experiences with accelerated reproductive development (Cameron et al., 2008; Maestripieri, 2005).

Accelerated reproductive development in harsh environments is often viewed as part of a "fast" life-history strategy that features reduced investment in long-term pair bonding and parenting (Belsky et al., 1991; Quinlan, 2007), increased investment in short-term mating (Belsky, 2012; Chisholm, 1993; Ellis et al., 2009), and increased risk taking (Frankenhuis \& Del Giudice, 2012), including aggression, status competition, and antisocial behavior (Belsky, Schlomer, \& Ellis, 2012; Simpson, Griskevicius, Kuo, Sung, \& Collins, 2012).

\section{DEVELOPMENTAL EFFECTS OF EARLY-LIFE UNPREDICTABILITY}

Until recently, unpredictability was lumped together with harshness (for discussion, see Brumbach, Figueredo, \& Ellis, 2009; Ellis et al., 2009; see also Baram et al., 2012). However, these dimensions are different (e.g., environments can be predictably harsh) and may require different responses. For instance, both unpredictability and harshness may shift individuals toward earlier reproduction, but a combination of the two may do so at a faster rate because mean life expectancy is low and individuals could die well before reaching this mean age if the environmental state worsens.

Studies have found that harsh and unpredictable environments during childhood are independently associated with "fast" life histories, such as earlier and more frequent sexual behavior, younger age at first conception, more aggressive and delinquent behaviors, and reduced health (Belsky et al., 2012; Brumbach et al., 2009; Nettle et al., 2011; Simpson et al., 2012). However, interpretations of these independent associations must remain tentative because measures of early-life harshness (e.g., low socioeconomic status, exposure to violence) and unpredictability (e.g., residential changes, family disruptions) may capture different components of harshness - each contributing to the mean level of harshness - rather than unpredictability - that is, variability in the level of harshness over time- per se (Nettle, Frankenhuis, \& Rickard, 2012).

\section{From Observation to Estimation}

The studies mentioned earlier measured unpredictability based on indices of changes or inconsistency in childhood environments. They did not address how children transform these experiences into cognitive estimates of environmental harshness and unpredictability. Research has examined the impact of early unpredictability on the development of infants' stress response (Cameron et al., 2008; Ellis, 2004), attachment styles (Bigelow et al., 2010; de Wolff \& van IJzendoorn, 1997; Gergely, Koós, \& Watson, 2010; Watson, 2001), expectations about maternal sensitivity (Johnson et al., 2010), mental schemas of unpredictability (Hill, Jenkins, \& Farmer, 2008; Ross \& Hill, 2002), and adults' cognitive responses to mortality primes (Griskevicius, 
Delton, Robertson, \& Tybur, 2011). Yet, we do not know how such schemas are constructed from earlier experiences.

\section{CONTINGENCY ANALYSIS}

Environmental predictability can be estimated using conditional probabilities (Gergely \& Watson, 1999; Maier \& Seligman, 1976; Shanks, 1995; Tarabulsy et al., 1996; Watson, 1967, 1979, 2001). For instance, an infant might observe that his coos are often followed by his mother smiling and use this knowledge to control his environment (e.g., elicit rewarding smiles). The probability of a given event (in this case, an action, cooing) resulting in a particular outcome (in this case, smiling) is called a prospective probability.

By themselves, prospective probabilities tell only half the story. What matters, too, is the likelihood that a given outcome (e.g., mother smiling) is preceded by a particular event (e.g., baby cooing) - a retrospective probability. Consider an example in which a mother always smiles (outcome) when her baby coos (action; prospective probability $=1$ ), but smiles equally often for reasons unrelated to her baby's actions (retrospective $p=.5$; see Figure 1, panel a). In this case, the child will be limited in his ability to control when his mother will smile. An imbalance might occur in the opposite direction as well: A mother might smile in response to her baby's coos half of the time (prospective $p=.5$ ), but all her smiles might be preceded by her baby cooing (retrospective $p=1$; see Figure 1, panel b). This reduced prospective probability also limits the child's control over his mother's smile.

Young infants are able to detect entities that respond contingently to their actions (e.g., their mother smiling in response to
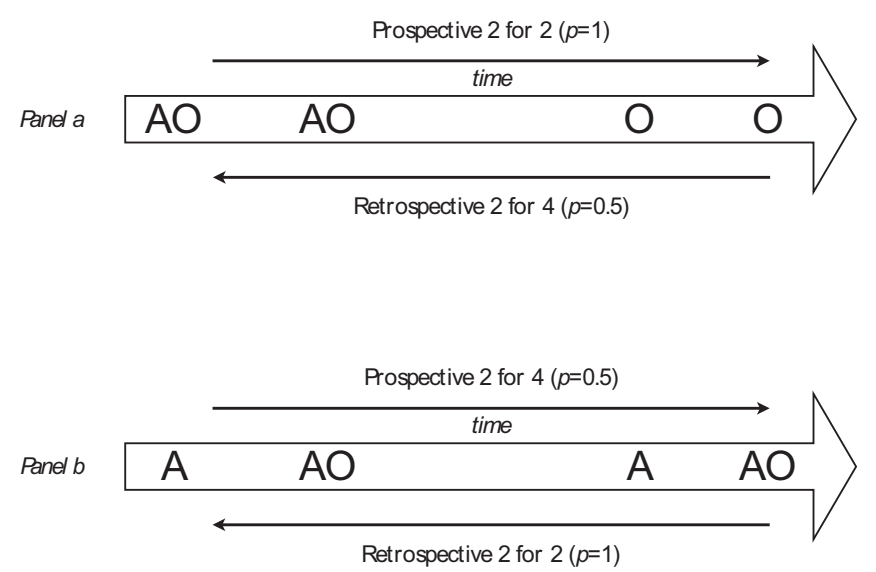

Figure 1. These panels illustrate imbalances that might occur between prospective and retrospective conditional probabilities. The prospective conditional is the probability of an outcome (denoted by " $O$ ": e.g., mother smiling) given an event, in this case, an action (denoted by "A": e.g., baby cooing). The retrospective conditional is the probability of the event, in this case, an action (A), preceding the outcome $(0)$. Imbalances of either kind may induce an "active learning" process in the child, in which he strives to (maximally) reduce his uncertainty about the true level of contingency (Butko \& Movellan, 2010) - a key component of environmental predictability. This figure was designed after Watson (2001). their cooing) and use this knowledge to control their immediate environment (Butko \& Movellan, 2010; Gergely \& Watson, 1999; Tarabulsy et al., 1996; Watson, 1967, 1979, 2001). We propose that infants also use their contingency experiences to guide their long-term development.

\section{Predictability and Control}

In the relation between unpredictability and control, control is one form of predictability, because one can safely predict the outcome of one's action if one has control (see also Brumbach et al., 2009). However, the loss of control does not necessarily imply that one cannot predict outcomes. Consider the case mentioned earlier in which an infant experiences a perfect prospective probability $(p=1)$ between his coo and his mother's smile, but at the same time, he experiences a reduced retrospective probability $(p=.5)$ because his mother sometimes smiles when he does not coo. The mother's smiles in the absence of the infant's coos still might be predictable if the infant can perceive their causes, for example, hearing his sister laugh. However, if the infant does not find the causes for those smiles he does not control, his mother's behavior is to that extent unpredictable. The relation between personal control and overall environmental predictability includes both controllable and uncontrollable events (see Figure 2).

Personal control is just one factor determining overall predictability. An environment might offer perfect control in that events occur anytime the individual performs a given action. However, depending on how often other causes bring about the same

\begin{tabular}{|c|c|c|c|c|c|}
\hline \multicolumn{2}{|c|}{} & \multicolumn{4}{|c|}{ Uncont rollable event s } \\
\cline { 2 - 6 } Personal cont rol & \multicolumn{2}{|c|}{ High R } & \multicolumn{2}{c|}{ Low R } \\
\cline { 2 - 6 } & High P & Low P & High P & Low P \\
\hline \multirow{2}{*}{ High R } & High P & High & High & High & High \\
\cline { 2 - 6 } & Low P & Low & Low & Low & Low \\
\hline \multirow{2}{*}{ Low R } & High P & High & Medium & Medium & Medium-Low \\
\cline { 2 - 6 } & Low P & Medium & Medium-Low & Medium-Low & Low \\
\hline
\end{tabular}

Figure 2. Environmental predictability depends on (a) an individual's control over the environment, and (b) his or her ability to determine causes of events he or she cannot control. In this table, "High $\mathrm{P}$ " denotes a high prospective conditional; "Low P," a low prospective conditional; "High R," a high retrospective conditional; and "Low R," a low retrospective conditional. If the retrospective conditional of personal control is high, very few outcomes occur without being preceded by the act; therefore, overall predictability will be that provided by personal control. If the retrospective conditional of personal control is low, however, overall predictability will depend on a combination of both personal control and analysis of external determinants of the outcome. 
events and whether or not the individual perceives those causes, overall predictability of these events can be high or low.

\section{EARLY CONTINGENCY EXPERIENCES ARE INFORMATIVE}

Contingency analyses of early experiences can provide information about the current environmental state. In many species, including humans, parents tend to produce many offspring when environments are harsh and/or unpredictable (Promislow \& Harvey, 1990; Stearns, 1992). This is adaptive in harsh conditions because any offspring might die young (Placek \& Quinlan, 2012) and in unpredictable conditions because even if conditions are not harsh at the moment (they might be), this could change (Ellis et al., 2009). Having more offspring implies less investment in any given offspring (Quinlan, 2007). Therefore, receiving low-quality care provides information about environmental harshness and/or predictability. Thus, children might use the extent of care they receive as a cue to guide their development (e.g., accelerating maturation; Belsky, 2012; Belsky et al., 1991; Chisholm, 1993; Ellis, 2004).

How are levels of parental investment reflected in the contingency experiences of infants? We restrict our treatment to the prospective and retrospective probabilities of rewards (i.e., fitness-enhancing experiences), leaving discussion of nonrewarding experiences (e.g., punishments) for future study.

\section{Contingency Profiles}

When she has many children, a mother's attention is divided among them, so she might miss instances of any particular infant's behavior. Moreover, if she does detect an infant's behavior (e.g., a request for food), she might withhold a response, prioritizing care for other offspring or engaging in activities not directly related to parenting (e.g., acquiring resources). From an infant's perspective, these factors lower the prospective conditional (P). We also expect the mother's probability of a noncontingent action (an act uncontrolled by the infant's behavior) toward any particular infant to be lower-that is, if the mother engages a child, this will more often be child initiated - raising, from the infant's viewpoint, the retrospective conditional (R). So, when the retrospective conditional is high relative to the prospective conditional (i.e., the $\mathrm{P} / \mathrm{R}$ ratio is small), this might serve as a cue to a harsh and/or unpredictable environment, inducing a "fast" life history in the child.

With a smaller family, we expect the opposite pattern. A mother's attention is divided among fewer infants and there are fewer extrafamilial stressors (e.g., scarcity of resources), resulting in her missing fewer of her infants' behaviors and responding more frequently to detected behaviors. From an infant's viewpoint, the prospective conditional will rise. Low-stress conditions might also raise a mother's willingness to provide noncontingent rewards - child-directed rewards not initiated by the child - lowering the retrospective conditional. So, when the prospective conditional is high relative to the retrospective conditional (i.e., the $P / R$ ratio is large), this might serve as a cue to a safe and predictable environment, inducing a "slow" life history.

In both scenarios, infants have limited control: in the harsh and/or unpredictable scenario due to a low prospective conditional, in the safe and predictable scenario due to a low retrospective conditional. Yet we expect very different developmental effects because the first scenario (low prospective, high retrospective) entails less parental investment than the second (high prospective, low retrospective).

\section{Separately Estimating Harshness and Predictability}

Because parents tend to produce larger families in harsh and/or unpredictable environments, offspring cannot distinguish between these based on experiencing a low P/R. However, parents face extrafamilial stressors as well (e.g., scarcity of resources, predators, disease). In environments that are predictably harsh, these will be stably present, resulting in a consistently low $\mathrm{P} / \mathrm{R}$. In unpredictable environments, extrafamilial stressors will fluctuate over time, influencing parental investment: In good times, parents can invest more (increasing $\mathrm{P} / \mathrm{R}$ ), and in bad times, less (lowering $\mathrm{P} / \mathrm{R})$. As a result, offspring will experience fluctuations in $\mathrm{P} / \mathrm{R}$, indicating environmental unpredictability.

In unpredictable environments, parents still produce large families and their investments in offspring are on average lower than that of parents in environments that are stably safe and supportive. However, because exposure to extrafamilial stressors fluctuates, the P/R experienced by offspring will vary, too.

\section{CONCLUSIONS}

Our article connects two literatures: evolutionary developmental psychology and contingency analysis. Environmental stressors affect parental investment (e.g., responsiveness), which then shapes children's development (e.g., attachment styles) into adolescence and adulthood (Belsky, 2012; Belsky et al., 1991; Ellis et al., 2009; Quinlan, 2007). Although research has detailed mediating physiological pathways (Cameron et al., 2008; Ellis, 2004), it has not specified how children transform experiences with their parents' and others' responsiveness into cognitive estimates about environmental harshness and unpredictability. Here, we argue that children use contingency analysis to develop these estimates.

We propose that both harshness and unpredictable environments lower the $\mathrm{P} / \mathrm{R}$ experienced by offspring, compared to stably safe environments (due to parents spreading out their investments). In addition, in unpredictable environments, offspring experience fluctuations in $\mathrm{P} / \mathrm{R}$ (associated with fluctuations in extrafamilial stressors), which they do not experience in stably harsh environments. Children estimate harshness via low $\mathrm{P} / \mathrm{R}$ and unpredictability via a combination of low $\mathrm{P} / \mathrm{R}$ and fluctuations in P/R. To stimulate testing of these ideas, we advance six empirical predictions - the first four of which are summarized in Figure 3: 


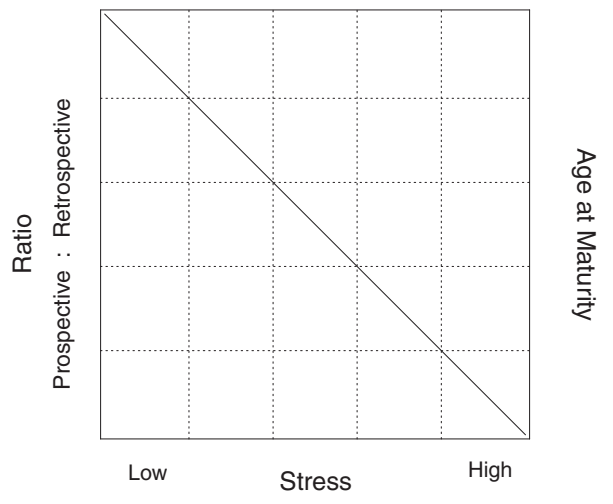

Figure 3. The horizontal axis depicts the level of environmental stress in early life (harshness and/or unpredictability). The left vertical axis depicts the ratio of the prospective over the retrospective conditional $(P / R)$. The right vertical axis depicts the age at reaching maturity. We predict that high-stress conditions will lower the prospective conditional and raise the retrospective conditional, reducing the $\mathrm{P} / \mathrm{R}$, accelerating maturation (resulting in younger age at maturity). Low-stress conditions will raise the prospective conditional and lower the retrospective conditional, increasing the $P / R$, decelerating maturation (resulting in older age at maturity). The diagonal line indicates a monotonic (although not necessarily linear) relationship.

1. In high-stress (harsh and/or unpredictable) conditions, infants' social contingency experiences will be characterized by a small $\mathrm{P} / \mathrm{R}$.

2. A small P/R will cause, hence be associated with, faster maturation.

3. In low-stress (safe and predictable) conditions, infants' social contingency experiences will be characterized by a large $\mathrm{P} / \mathrm{R}$.

4. A large $P / R$ will cause, hence be associated with, slower maturation.

5. In unpredictable environments, infants will experience variable P/Rs.

6. A variable $\mathrm{P} / \mathrm{R}$ will cause, hence be associated with, faster maturation, above and beyond the effects of mean levels of adversity.

Although these predictions are general, children vary in their susceptibility to environmental influences and learning strategies (Belsky, 2012; Frankenhuis \& Panchanathan, 2011a; Sih \& Del Giudice, 2012). Could variation in the ability to learn contingencies contribute to perceptions of environmental predictability, with implications for the age at maturation? Individuals who better estimate true levels of contingencies (social and nonsocial) might perceive their environments as more predictable than individuals who are less proficient at this task. Hence, more proficient individuals might mature later than less proficient individuals, even if both develop in comparable physical and social environments.

\section{Final Thoughts}

In this article, we have not addressed how infants come to know which behaviors of others count as rewards or punishment, and how they become more effective at soliciting rewards and avoiding punishment (see Gergely \& Watson, 1999; Tarabulsy et al., 1996; Watson, 1967, 1979, 2001). We have also not addressed how infants learn which objects are social agentsand respond to them - and which ones are not. Computer scientists are examining this question, building computational models of the learning process by which infants reduce their uncertainty, as fast as possible, about the level of contingency objects display toward them (Butko \& Movellan, 2010).

Contingency analysis is a cognitive process by which infants and children can estimate environmental harshness and unpredictability. Harshness and unpredictability produce distinct contingency profiles (average $\mathrm{P} / \mathrm{R}$ vs. variability in $\mathrm{P} / \mathrm{R}$ ), and children can use these profiles to separately estimate each dimension. This view connects evolutionary developmental psychology's focus on long-term developmental outcomes (e.g., onset of menarche) being shaped by major life events (e.g., residential transitions) with the study of contingency analysis: the micro-analysis of the conditional probabilities of actions and outcomes infants experience. Most research of human life-history development has focused on children, not infants (for an exception, see Belsky et al., 2010). As a complement, contingency analysis can be used starting at birth, allowing the discovery of very early postnatal determinants of life-history strategy.

\section{REFERENCES}

Baram, T. Z., Davis, E. P., Obenaus, A., Sandman, C. A., Small, S. L., Solodkin, A., et al. (2012). Fragmentation and unpredictability of early-life experience in mental disorders. American Journal of Psychiatry, 169, 907-915.

Belsky, J. (2012). The development of human reproductive strategies: Progress and prospects. Current Directions in Psychological Science, $21,310-316$.

Belsky, J., Houts, R. M., \& Fearon, R. M. P. (2010). Infant attachment security and the timing of puberty: Testing an evolutionary hypothesis. Psychological Science, 21, 1195-1201.

Belsky, J., Schlomer, G. L., \& Ellis, B. J. (2012). Beyond cumulative risk: Distinguishing harshness and unpredictability as determinants of parenting and early life history strategy. Developmental Psychology, 48, 662-673.

Belsky, J., Steinberg, L., \& Draper, P. (1991). Childhood experience, interpersonal development, and reproductive strategy: An evolutionary theory of socialization. Child Development, 62, $647-670$.

Bigelow, A. E., MacLean, K., Proctor, J., Myatt, T., Gillis, R., \& Power, M. (2010). Maternal sensitivity throughout infancy: Continuity and relation to attachment security. Infant Behavior and Development, $33,50-60$.

Brumbach, B. H., Figueredo, A. J., \& Ellis, B. J. (2009). Effects of harsh and unpredictable environments in adolescence on development of life history strategies: A longitudinal test of an evolutionary model. Human Nature, 20, 25-51.

Butko, N. J., \& Movellan, J. R. (2010). Detecting contingencies: An infomax approach. Neural Networks, 23, 973-984.

Cameron, N. M., Del Corpo, A., Diorio, J., McAllister, K., Sharma, S., \& Meaney, M. J. (2008). Maternal programming of sexual behavior 
and hypothalamic-pituitary-gonadal function in the female rat. PLOS ONE, 3, e2210.

Chisholm, J. S. (1993). Death, hope, and sex: Life-history theory and the development of reproductive strategies. Current Anthropology, 34, $1-24$.

Chisholm, J. S., Quinlivan, J. A., Petersen, R. W., \& Coall, D. (2005). Early stress predicts age at menarche and first birth, adult attachment and expected lifespan. Human Nature, 16, 233-265.

de Wolff, M. S., \& van IJzendoorn, M. H. (1997). Sensitivity and attachment: A meta-analysis on parental antecedents of infant attachment. Child Development, 68, 571-591.

Ellis, B. J. (2004). Timing of pubertal maturation in girls: An integrated life history approach. Psychological Bulletin, 130, 920-958.

Ellis, B. J., Figueredo, A. J., Brumbach, B. H., \& Schlomer, G. L. (2009). Fundamental dimensions of environmental risk: The impact of harsh versus unpredictable environments on the evolution and development of life history strategies. Human Nature, 20, 204268.

Ellison, P. T. (2001). On fertile ground: A natural history of human reproduction. Cambridge, MA: Harvard University Press.

Frankenhuis, W. E., \& Del Giudice, M. (2012). When do adaptive developmental mechanisms yield maladaptive outcomes? Developmental Psychology, 48, 628-642.

Frankenhuis, W. E., \& Panchanathan, K. (2011a). Individual differences in developmental plasticity may result from stochastic sampling. Perspectives on Psychological Science, 6, 336-347.

Frankenhuis, W. E., \& Panchanathan, K. (2011b). Balancing sampling and specialization: An adaptationist model of incremental development. Proceedings of the Royal Society B, 278, 3558-3565.

Gergely, G., Koós, O., \& Watson, J. S. (2010). Contingent parental reactivity in early socio-emotional development. In T. Fuchs, H. C. Sattel, \& P. Henningsen (Eds.), The embodied self: Dimensions, coherence and disorders (pp. 141-169). Stuttgart, Germany: Schattauer.

Gergely, G., \& Watson, J. S. (1999). Early social-emotional development: Contingency perception and the social biofeedback model. In P. Rochat (Ed.), Early social cognition (pp. 101-136). Mahwah, NJ: Erlbaum.

Griskevicius, V., Delton, A. W., Robertson, T. E., \& Tybur, J. M. (2011). The environmental contingency of life history strategies: Influences of mortality and socioeconomic status on reproductive timing. Journal of Personality and Social Psychology, 100, 241254.

Hill, E. M., Jenkins, J., \& Farmer, L. (2008). Environmental unpredictability, future discounting, and risk taking. Journal of Socio-Economics, 37, 1381-1396.

Johnson, S. C., Dweck, C., Chen, F. S., Ok., S. J., Stern, H. L., \& Barth, M. E. (2010). At the intersection of social and cognitive development: Internal working models of attachment in infancy. Journal of Cognitive Science, 34, 807-825.

Maestripieri, D. (2005). Effects of early experience on female behavioural and reproductive development in rhesus macaques. Proceedings of the Royal Society B-Biological Sciences, 272, 1243-1248.

Maestripieri, D., Roney, J. R., De Bias, N., Durante, K. M., \& Spaepen, G. M. (2004). Father absence, menarche and interest in infants among adolescent girls. Developmental Science, 7, 560-566.
Maier, S. F., \& Seligman, M. E. P. (1976). Learned helplessness: Theory and evidence. Journal of Experimental Psychology: General, 105, $3-46$.

Nettle, D., Coall, D. A., \& Dickins, T. E. (2011). Early life conditions and age at first pregnancy in British women. Proceedings of the Royal Society B: Biological Sciences, 278, 1721-1727.

Nettle, D., Frankenhuis, W. E., \& Rickard, I. J. (2012). The adaptive basis of psychosocial acceleration theory. Developmental Psychology, 48, 718-721.

Painter, R. C., Westendorp, R. G., de Rooij, S. R., Osmond, C., Barker, D. J., \& Roseboom, T. J. (2008). Increased reproductive success of women after prenatal undernutrition. Human Reproduction, 23, 2591-2595.

Pesonen, A.-K., Räikkönen, K., Heinonen, K., Kajantie, E., Forsén, T., \& Eriksson, J. G. (2008). Reproductive traits following a parentchild separation trauma during childhood: A natural experiment during World War II. American Journal of Human Biology, 20, 345-351.

Placek, C. D., \& Quinlan, R. J. (2012). Adolescent fertility and risky environments: A population-level perspective across the lifespan. Proceedings of the Royal Society B, 279, 4003-4008.

Promislow, D. E. L., \& Harvey, P. H. (1990). Living fast and dying young: A comparative analysis of life-history variation among mammals. Journal of Zoology, 220, 417-437.

Quinlan, R. J. (2007). Human parental effort and environmental risk. Proceedings of the Royal Society B, 274, 121-125.

Ross, L. T., \& Hill, E. M. (2002). Childhood unpredictability, schemas for unpredictability, and risk taking. Social Behavior and Personality, 30, 453-474.

Shanks, D. R. (1995). Is human learning rational? Quarterly Journal of Experimental Psychology: Human Experimental Psychology, 48, 257-279.

Sih, A., \& Del Giudice, M. (2012). Linking behavioural syndromes and cognition: A behavioural ecology perspective. Philosophical Transactions of the Royal Society B, 367, 2762-2772.

Simpson, J. A., Griskevicius, V., Kuo, S. I.-C., Sung, S., \& Collins, W. A. (2012). Evolution, stress, and sensitive periods: The influence of unpredictability in early versus late childhood on sex and risky behavior. Developmental Psychology, 48, 674-686.

Stearns, S. C. (1992). The evolution of life histories. Oxford, England: Oxford University Press.

Tarabulsy, G. M., Tessier, R., \& Kappas, A. (1996). Contingency detection and the contingent organization of behavior in interactions: Implications for socioemotional development in infancy. Psychological Bulletin, 120, 25-41.

Tither, J. M., \& Ellis, B. J. (2008). Impact of fathers on daughters' age at menarche: A genetically and environmentally controlled sibling study. Developmental Psychology, 44, 1409-1420.

Watson, J. S. (1967). Memory and "contingency analysis" in infant learning. Merrill-Palmer Quarterly, 13, 55- 76.

Watson, J. S. (1979). Perception of contingency as a determinant of social responsiveness. In E. B. Thoman (Ed.), Origins of the infant's social responsiveness (pp. 33-64). Hillsdale, NJ: Erlbaum.

Watson, J. S. (2001). Contingency perception and misperception in infancy: Some potential implications for attachment. Bulletin of the Menninger Clinic, 65, 296-320. 